\title{
Predictive value of adipose to muscle area ratio based on MRI at knee joint for postoperative functional outcomes in elderly osteoarthritis patients following total knee arthroplasty
}

Guanglei Zhao ${ }^{\dagger}$, Changquan Liu ${ }^{\dagger}$, Kangming Chen, Feiyan Chen, Jinyang Lyu, Jie Chen, Jingsheng Shi, Gangyong Huang, Yibing Wei, Siqun Wang and Jun Xia

\begin{abstract}
Background: The current research used a new index-adipose to muscle area ratio (AMR) - to measure fatness compared with body mass index (BMI) in elderly osteoarthritis (OA) patients following total knee arthroplasty. Our study aimed to test the relationship between the two indexes (AMR and BMI) and to examine whether AMR was a predictive factor of patient-reported outcome measures (PROMS) for elderly OA patients following total knee arthroplasty (TKA).

Methods: The retrospective data of 78 OA patients (older than 60 years) following TKA was included in our study. Clinical features of patients included age, BMI, sex, AMR, side of the implant, time of follow-up, complications, the Knee Society Score (KSS score), and the Hospital for Special Surgery knee score (HSS score). The area of adipose tissue and muscle tissue was measured on the cross section (supra-patella, midline of the patella, joint line of the knee) of the knee magnetic resonance imaging (MRI). AMR was calculated as the average of adipose to muscle area ratio at the three levels. The Pearson correlation analysis, simple linear regression, and multiple linear regression were used to study the relationship between BMI, AMR, and PROMS (KSS total-post score and HSS-post score) in the study.

Results: Of all patients, the mean ( \pm standard deviations (SD)) of age was $67.78 \pm 4.91$ years. For BMI and AMR, the mean $( \pm$ SD) were $26.90 \pm 2.11$ and $2.36 \pm 0.69$, respectively. In Pearson correlation analysis, BMI had a good correlation with AMR $(r=0.56, p=0.000)$, and AMR $(r=-0.37, p=0.001$, HSS-post score; $r=-0.43, p=0.000$, KSS total-post score) had better correlations with PROMS postoperatively compared with BMI $(r=-0.27, p=0.019$, HSSpost score; $r=-0.33, p=0.003$, KSS total-post score). In multivariate linear regression analysis, AMR was negatively correlated with KSS total-post score as well as HSS-post score, while BMI was not. As for patients with complications, AMR values were between the 3rd quartile and 4th quartile of the AMR value in the entire study (Continued on next page)
\end{abstract}

\footnotetext{
* Correspondence: hudbt17089@gmail.com

${ }^{\dagger}$ Guanglei Zhao and Changquan Liu contributed equally to this work. Department of Orthopedics, Huashan Hospital, Fudan University, Shanghai 200040, China
}

C C The Author(s). 2020 Open Access This article is licensed under a Creative Commons Attribution 4.0 International License, which permits use, sharing, adaptation, distribution and reproduction in any medium or format, as long as you give appropriate credit to the original author(s) and the source, provide a link to the Creative Commons licence, and indicate if changes were made. The images or other third party material in this article are included in the article's Creative Commons licence, unless indicated otherwise in a credit line to the material. If material is not included in the article's Creative Commons licence and your intended use is not permitted by statutory regulation or exceeds the permitted use, you will need to obtain permission directly from the copyright holder. To view a copy of this licence, visit http://creativecommons.org/licenses/by/4.0/. The Creative Commons Public Domain Dedication waiver (http://creativecommons.org/publicdomain/zero/1.0/) applies to the data made available in this article, unless otherwise stated in a credit line to the data. 
(Continued from previous page)

cohort.

Conclusions: In this study, the new obesity evaluation indicator-AMR, which was well related with BMI, was

found to be a predictor of PROMS (KSS total-post score and HSS-post score) in elderly OA patients following TKA.

Keywords: Adipose to muscle area ratio (AMR), Body mass index (BMI), Osteoarthritis (OA), Predictor, Patientreported outcome measures (PROMS), Total knee arthroplasty (TKA)

\section{Introduction}

Obesity is a worldwide health problem and has almost tripled since 1975 throughout the world [1]. According to a 2016 data, there were 43.2 million men and 46.4 million women in China, and the number of obese people exceeded that of the USA, ranking first in the world [2]. As a health problem, obesity is more common in elderly people and is associated with many diseases, including osteoarthritis (OA) [3-6].

As we all know, body mass index (BMI) is currently the common indicator used to evaluate obesity and BMI $\geq 30 \mathrm{~kg} / \mathrm{m}^{2}$ is defined as obesity [7]. There were many studies showing that obesity (high BMI) was associated with poor outcomes of elderly OA patients following total knee arthroplasty (TKA) [8-12]. Two metaanalyses showed that the risk of both shallow and deep infections was higher in obese patients who have underwent TKA than that of non-obese patients $[8,9]$. Another meta-analysis made by Sun et al. [10] proposed that high BMI influenced postoperative functional outcomes and enhanced the risk of complications in patients following TKA. However, using BMI alone to assess the prognosis in elderly OA patients may have certain limitations. BMI is not an absolute health indicator because it does not provide a specific proportion of body composition such as the muscle, fat, and bones [13]. Sarcopenic obesity, often seen in elderly patients, in which total muscle mass, muscle strength, and physical functional decrease, could not be well represented through BMI $[14,15]$. Sometimes barely relying on BMI may lead to classification bias and delay surgery for obese patients [16]. Patients with central obesity may have thin limbs and they are likely to have a good prognosis after TKA. However, such patients usually have higher BMI [17]. Meanwhile, there were studies reporting that no significant correlation was found between BMI and functional outcomes in elderly OA patients after TKA $[18,19]$.

To address the limitations of BMI in assessing prognosis in elderly OA patients following TKA, there were many researches using other indicators (fat mass, skeletal muscle mass, subcutaneous fat thickness, knee mass index, and so on) to better provide body composition in obese patients and study their relationship with patients' prognosis $[17,20,21]$. It was worth mentioning that, in a recent study, Dai et al. [22] proposed a new indicator-adipose to muscle area ratio (AMR) - to assess fatness and they concluded that compared with BMI, AMR at the knee joint showed better predictive ability in predicting functional outcomes for patients following meniscectomy.

Obese patients were more susceptible to OA than those who were not, and TKA was an end-stage treatment for elderly OA patients [3, 23, 24]. AMR proposed by Dai et al. [22] was a new index to assess obesity and showed good predictive ability of postoperative outcomes in patients undergoing meniscectomy. There was no other study using AMR to assess obesity and examine its predictive ability of prognosis in elderly OA patients undergoing TKA. Our research is the first study trying to use this new index to evaluate obesity and test its predictive ability of postoperative outcomes in elderly OA patients after TKA. And we want to answer three questions through our research: (1) Is AMR at knee joint related to BMI used to evaluate obesity? (2) Can AMR at knee joint be used as a predictor of postoperative reported outcomes (the Knee Society Score (KSS score) and the Hospital for Special Surgery knee score (HSS score)) for elderly OA patients following TKA, and is it better than BMI? (3) Is AMR a predictor in predicting complications of elderly OA patients undergoing TKA?

\section{Methods \\ Study design and study cohort}

We retrospectively analyzed the total knee replacement cases $(n=656)$ in our hospital (Huashan Hospital, Fudan University) from 2013 to 2017. Among all the patients, we only retained those with knee magnetic resonance imaging (MRI) before surgery $(n=127)$. Then we excluded 49 patients according to the exclusion criteria: (1) patients with bilateral TKA surgery $(n=12),(2)$ patients not diagnosed with knee OA $(n=11)$, (3) Deyo score greater than 2 points $(n=15)$, (4) patients younger than 60 years $(n=6)$, and (5) lost to follow-up $(n=5)$. Finally, we included a total of 78 patients in our study. Figure 1 shows the screening process for the study. This study was approved by the institutional review board of Huashan Hospital, Fudan University.

The clinical features included age, BMI, sex, adipose to muscle area ratio (AMR), side of the implant, time of 


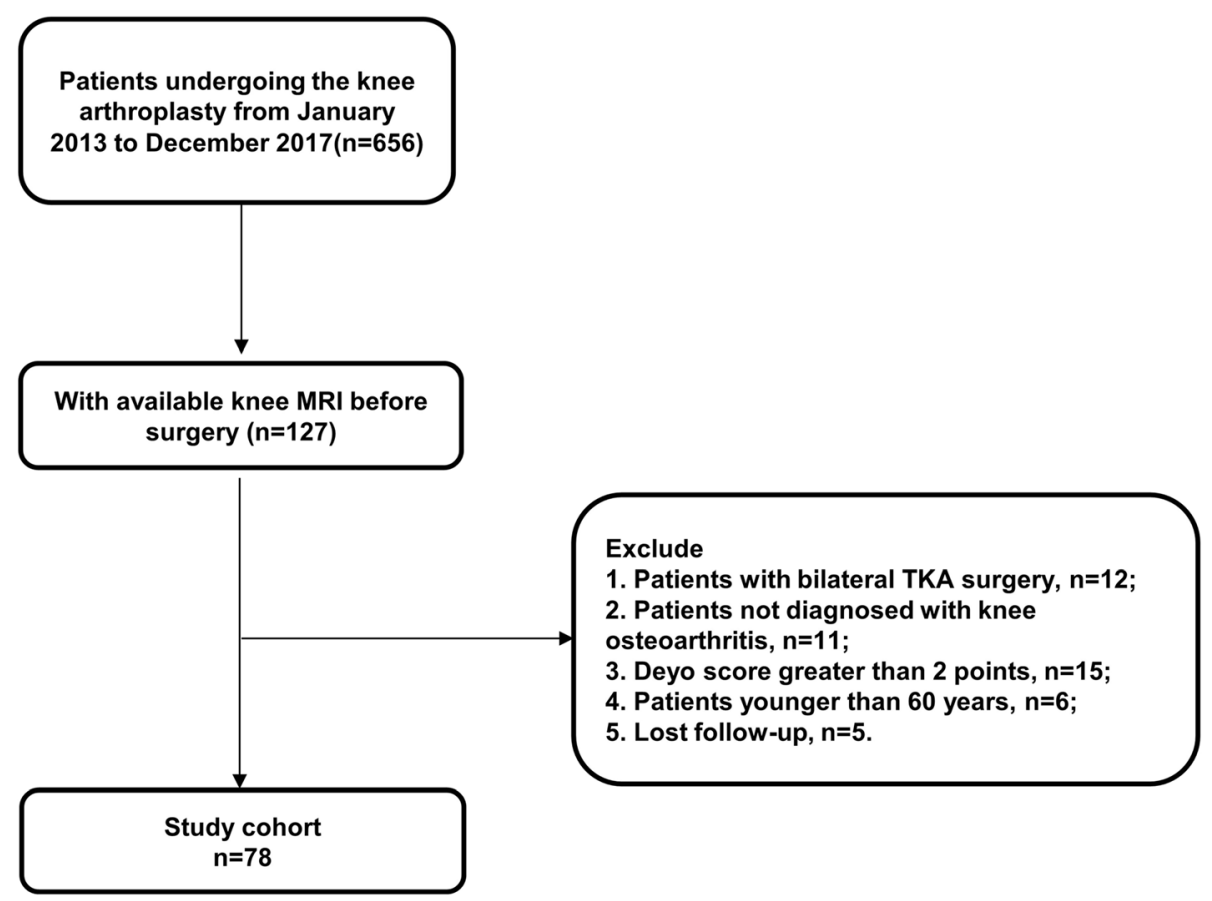

Fig. 1 Flow diagram of the selecting process in the study

follow-up, and complications. The KSS score and the HSS score were used in our study to assess the functional outcome of patients. The KSS score, which is a questionnaire designed to evaluate the knee of patients, includes two parts: a knee score and a function score, both of which score from 0 to 100 , with high scores representing better status [25]. The HSS score, which ranged from 0 to 100 points, contained a total of 7 parts (pain-30 points, functional-22 points, activity-18 points, muscle strength -10 points, knee flexion -10 points, stability -10 points, and point reduction) [26, 27]. Both the KSS score and HSS score were recorded before surgery (KSS-pre and HSS-pre) and at the most recent follow-up (KSS-post and HSS-post).

\section{Measurements of AMR}

A method used to calculate AMR at the level of the joint line was introduced in a previous study by Dai et al. [22]. In our study, the area of adipose tissue and muscle tissue was measured on the cross section of the knee MRI (supra-patella, midline of the patella, joint line of the knee). The red part was the area of the joint cavity $(\mathrm{R})$, the yellow part was the area of the muscle tissue $(\mathrm{Y})$, the part circled by the blue line was the area of the entire cross section (B), and the area of the adipose tissue (A) was calculated by subtracting $\mathrm{Y}$ and $\mathrm{R}$ from $\mathrm{B}$. $\mathrm{AMR}=\mathrm{A} / \mathrm{Y}$ (single level), $\mathrm{AMR}$ at the level of the supra-patella was recorded as AMR up, AMR at the level of the midline of the patella-AMR middle, and AMR at the joint line level-AMR down. The average of the AMRs at the three levels was taken as the final AMR (recorded as AMR in our study) (Fig. 2). Area measurement was done with the software ImageJ. Good inter-observer reliability of AMR measurements was shown between two observers using the intraclass correlation coefficient (ICC) $($ ICC-AMR up $=0.982$, ICC-AMR middle $=0.991$, ICC-AMR down $=0.984)$.

\section{Complications}

Composite complications after TKA included superficial incisional surgical site infection (SSI), deep incisional SSI, organ space SSI, wound disruption, pneumonia, unplanned intubation, pulmonary embolism, on ventilator $>48 \mathrm{~h}$, progressive renal insufficiency, acute renal failure, urinary tract infection, stroke/cerebrovascular accident (CVA), coma $>24 \mathrm{~h}$, peripheral nerve injury, dislocation, pseudoaneurysm, cardiac arrest, myocardial infarction, prosthesis failure, implant loosening, deep vein thrombosis (DVT), high metal ion levels of blood, sepsis, septic shock, and so on [28-35].

\section{Statistical analysis}

The continuous variables (age, BMI, AMR, time of follow-up, KSS score, and HSS score) were presented as means and standard deviations (SD), while the categorical variables (sex, side of the implant) were given as frequencies and percentages (\%). 


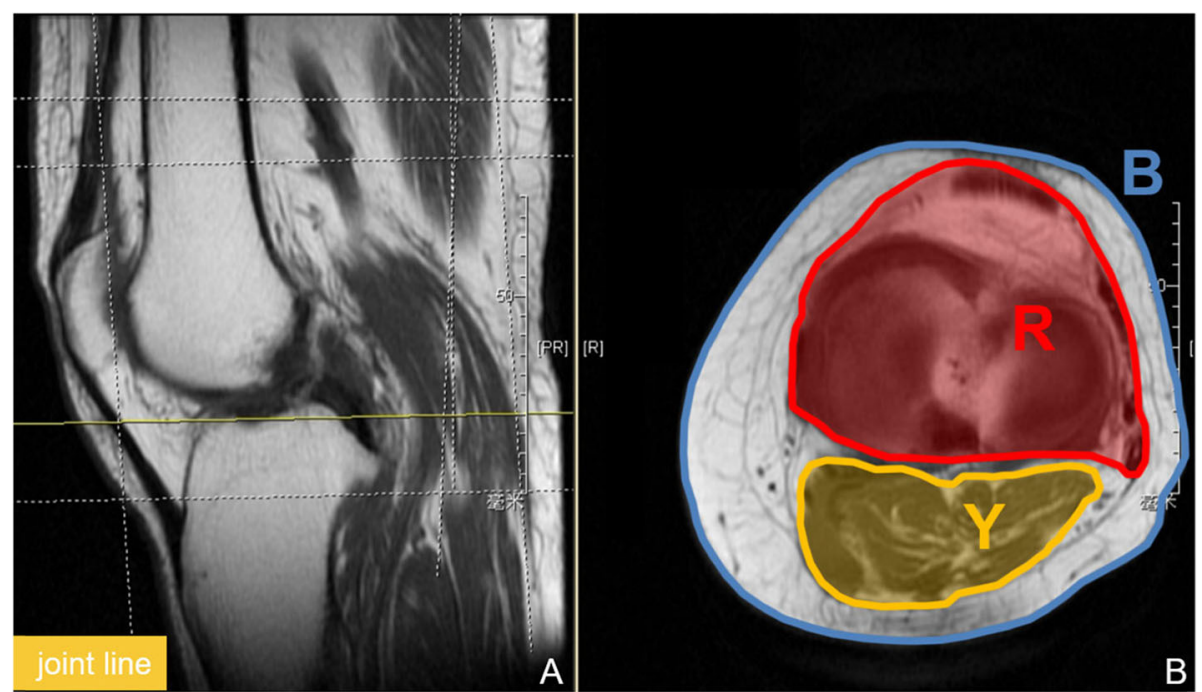

Fig. 2 a, b Schema of AMR measurement on the cross section of the knee MRI at the level of the knee joint line. a Sagittal plane. $\mathbf{b}$ Cross section. The area of the joint cavity (R), the muscle tissue $(Y)$, and the entire cross section (B)

Student's $t$ test was carried out to examine the discrepancy of AMR value in different genders and sides of the implant. The Pearson correlation analyses were done among those variables (age, AMR, BMI, HSS-pre, and KSS total-pre). Correlations between AMR and KSS total-post, AMR and HSS-post, BMI and KSS total-post, and BMI and HSS-post were also analyzed using the same method. The correlation coefficient $(r)$ was used to measure the correlation between two variables. Positive values represented positive correlations while negative correlations were revealed by negative values. The larger the absolute value, the stronger the correlation $(|r|=1$ represents a linear relationship). In simple linear regression analysis, variables KSS total-post and HSS-post score were defined as dependent variables, while age, BMI, sex, AMR, side of the implant, KSS total-pre score, and HSS-pre score were analyzed as independent variables. Variables $(p<0.1)$ in univariate analysis were further analyzed in multivariate linear regression analysis.

Student's $t$ test, the Pearson correlation analysis, simple linear regression analysis, and multivariate linear regression analysis were conducted in SPSS 24.0. All analyses with $p<0.05$ (two-sided) were considered statistically significant.

\section{Results}

In our study, a total of 78 patients were included. Of all patients, the mean $( \pm \mathrm{SD})$ of age was $67.78 \pm 4.91$ years, the BMI $26.90 \pm 2.11 \mathrm{~kg} / \mathrm{m}^{2}$, the AMR $2.36 \pm 0.69$, the follow-up time $31.91 \pm 6.49$ months, the KSS total-pre score $105.68 \pm 20.84$, the KSS total-post score $181.51 \pm$ 11.05 , the HSS-pre score $47.77 \pm 8.34$, and the HSS-post score $91.74 \pm 4.77$. In this study, 43 patients were female and 35 patients were male. The surgical sites of $43 \mathrm{pa}$ tients were the right knee and the remaining 35 patients were the left knee. A total of three patients had postoperative complications: one had a superficial incisional surgical site infection (SSI), and another two had a urine tract infection and a prosthesis failure (periprosthetic infection), respectively (Tables 1 and 5). As for AMR values, no significant difference was found between male and female, not in sides of the implant, either.

In Pearson correlation analysis, significant correlations between AMR and BMI $(r=0.56, p=0.000)$, age and KSS total-pre $(r=0.230, p=0.042)$, and HSS-pre and KSS total-pre $(r=0.919, p=0.000)$ were shown. Meanwhile, AMR had stronger correlations with PROMS (KSS total-post and HSS-post score) compared with BMI (Table 2).

In the simple linear regression analysis of PROMS (KSS total-post score and HSS-post score), AMR ( $p=$ $0.000)$ and BMI $(p=0.003)$ were negatively related with the KSS total-post score. It was the same two variables (AMR, $p=0.001$; BMI, $p=0.019$ ) showing negative correlations with the HSS-post score. In further multivariate analysis, only AMR was investigated to have a significant relationship with the KSS total-post score $(p=0.007)$, as well as with the HSS-post score $(p=0.014)$, while BMI was not (Tables 3 and 4).

The interquartile range (IQR) of the AMR for the entire study cohort was $1.83-2.72$. In the study, we found that the AMR values of patients with complications were between 75 and $100 \%$ of the values of the entire study cohort (3rd quartile-4th quartile). The AMR values of patients with complications were 2.80 (superficial incisional surgical site infection (SSI)), 3.15 (urinary tract 
Table 1 Baseline characteristics

\begin{tabular}{|c|c|}
\hline Parameter & Continuous variables: mean $\pm \mathrm{SD}$; categorical variables: frequency (\%) \\
\hline Age (years) & $67.78 \pm 4.91$ \\
\hline BMI $\left(\mathrm{kg} / \mathrm{m}^{2}\right)$ & $26.90 \pm 2.11$ \\
\hline \multicolumn{2}{|l|}{ Sex } \\
\hline Male & $35(44.9 \%)$ \\
\hline Female & $43(55.1 \%)$ \\
\hline AMR & $2.36 \pm 0.69$ \\
\hline \multicolumn{2}{|l|}{ Side } \\
\hline Right & $43(55.1 \%)$ \\
\hline Left & $35(44.9 \%)$ \\
\hline Follow-up (months) & $31.91 \pm 6.49$ \\
\hline \multicolumn{2}{|l|}{ KSS score } \\
\hline KSS knee-pre & $49.14 \pm 9.92$ \\
\hline KSS function-pre & $56.54 \pm 11.47$ \\
\hline KSS total-pre & $105.68 \pm 20.84$ \\
\hline KSS knee-post & $89.91 \pm 5.23$ \\
\hline KSS function-post & $91.60 \pm 6.77$ \\
\hline KSS total-post & $181.51 \pm 11.05$ \\
\hline \multicolumn{2}{|l|}{ HSS score } \\
\hline HSS-pre & $47.77 \pm 8.34$ \\
\hline HSS-post & $91.74 \pm 4.77$ \\
\hline
\end{tabular}

SD standard deviation, BMI body mass index, AMR adipose to muscle area ratio, KSS score the Knee Society Score, HSS score the Hospital for Special Surgery knee score

infection), and 3.63 (prosthesis failure-periprosthetic infection), respectively (Table 5).

\section{Discussion}

In our research, we used a new indicator-AMR-which was well correlated with BMI to evaluate obesity in elderly OA patients following TKA. This indicator was found to be a predictor of PROMS (HSS-post score and KSS total-post score), while BMI was not. This illustrated that AMR in the knee joint was a stronger risk factor of poor functional outcomes more than BMI. Moreover, patients with complications were investigated with high AMR values (range: 3rd quartile to 4th quartile) in our study, which suggested that AMR might have a correlation with postoperative complications in elderly OA patients after TKA.

Table 2 Correlation analysis between AMR and BMI, AMR and KSS total-post, AMR and HSS-post, BMI and KSS total-post, and $\mathrm{BMI}$ and HSS-post

\begin{tabular}{|c|c|c|c|c|c|c|}
\hline & \multicolumn{2}{|l|}{ BMI } & \multicolumn{2}{|c|}{ KSS total-post } & \multicolumn{2}{|c|}{ HSS-post } \\
\hline & $r$ & $p$ & $r$ & $p$ & $r$ & $p$ \\
\hline$\overline{A M R}$ & 0.56 & 0.000 & -0.43 & 0.000 & -0.37 & 0.001 \\
\hline BMl & - & - & -0.33 & 0.003 & -0.27 & 0.019 \\
\hline
\end{tabular}

$B M I$ body mass index, $A M R$ adipose to muscle area ratio, $K S S$ score the Knee Society Score, HSS score the Hospital for Special Surgery knee score
BMI had many advantages in measuring obesity of elderly OA patients, such as ease of use and accurately defining obesity in most cases [13]. Many studies had showed there was a correlation between BMI and outcomes for elderly OA patients following TKA $[8,9,11$, 36]. $\mathrm{Xu}$ et al. [36] found that patients with higher BMI were more likely to have a smaller enhancement in functional outcome scores (Oxford Knee Score (OKS)). Giesinger et al. [11] found that BMI had a negative impact on functional outcome scores and satisfaction scores for patients following TKA. Although a lot of articles investigated that BMI negatively affected postoperative outcomes in elderly OA patients after TKA, there was no agreement on this issue $[18,19]$. Overgaard et al. [18] reported that the 1-year functional outcomes of patients following TKA were not influenced by BMI in a cohort of 3327 patients. Sveikata et al. [19] found no significant influence of BMI on postoperative functional outcome (OKS) for patients undergoing TKA. In a multivariate linear analysis of our study, no significant relationships between BMI and PROMS (KSS total-post score and HSS-post score) were investigated (Tables 3 and 4). These results showed the limitations of BMI to assess adiposity and evaluate the prognosis in elderly OA patients following TKA. The limitations of BMI were due to its own characteristics $[13,16]$. People with the same 
Table 3 Simple and multiple linear regression of HSS-post score

\begin{tabular}{|c|c|c|c|c|c|c|}
\hline & Simple & ression & & Multiple & gression & \\
\hline & $\beta$ & $95 \% \mathrm{Cl}$ & $p$ & $\beta$ & $95 \% \mathrm{Cl}$ & $p$ \\
\hline Age & 0.043 & -0.179 to 0.265 & n.s & & & \\
\hline BMI & -0.599 & -1.096 to -0.102 & 0.019 & -0.166 & -0.742 to 0.410 & n.s \\
\hline Sex & -0.361 & -2.551 to 1.828 & n.s & & & \\
\hline AMR & -2.587 & -4.054 to -1.120 & 0.001 & -2.223 & -3.990 to -0.455 & 0.014 \\
\hline Side & 0.727 & -1.458 to 2.912 & n.s & & & \\
\hline KSS total-pre & 0.046 & -0.005 to 0.097 & 0.078 & 0.018 & -0.105 to 0.141 & n.s \\
\hline HSS-pre & 0.115 & -0.013 to 0.243 & 0.078 & 0.060 & -0.248 to 0.368 & n.s \\
\hline
\end{tabular}

Cl confidence interval, n.s nonsignificant, BMI body mass index, AMR adipose to muscle area ratio, KSS score the Knee Society Score, HSS score the Hospital for Special Surgery knee score

BMI may have different organizational ratios. Using BMI alone to assess the postoperative outcomes in elderly OA patients following TKA ignored the effects of different body components on patients' prognosis, especially the muscle mass component. In contrast to BMI, AMR could better show the local distribution of muscle mass and adipose components in the knee joint. Meanwhile, the strength of quadriceps was related to the prognosis of patients after TKA. These might explain that AMR had stronger correlations with PROMS compared with BMI (Table 2) and could be used as a predictor of functional outcomes in elderly patients following TKA (Tables 3 and 4) in our research. To note, there were studies proposing that extremities of BMI might have better predictive ability of functional outcomes after surgery [22, 37]. Therefore, we speculated that AMR might be a supplement to BMI in body composition evaluation, especially in the assessment of local extremities.

Sarcopenic obesity was defined as a state in which obesity and sarcopenia co-occur. This term was described as total muscle mass, muscle strength, and physical functional decline often in the elderly $[14,15,38$, 39]. EWGSOP recommends using computed tomography $(\mathrm{CT})$, magnetic resonance imaging (MRI), dualenergy X-ray absorptiometry (DXA), and bioimpedance analysis (BIA) to measure muscle mass [14]. Therefore,
AMR measured through MRI can be regarded as one of sacropenia's evaluation methods. In recent studies, Babu et al. [15] found that the psoas-lumbar vertebral index (measured by CT images), an indicator of central sacropenia, had good predictive value for prosthetic joint infections (PJIs). Chang et al. [40] investigated that paraspinal muscle density (PSD) and skeletal muscle index (SMI) at L4 (an indicator of sacropenia) had negative effects on perioperative outcome for patients who had proximal femur fractures. Wagner et al. [41] discovered that larger psoas cross-sectional area (CSA) was a protective factor of degenerative spondylolisthesis. In line with them, we found AMR was an independent predictor of PROMS (KSS total-post score and HSS-post score) in elderly OA patients following TKA in our study (Tables 3 and 4). Meanwhile, the current use of MRI was becoming more and more widespread in process of diagnosis and treatment [42-45]. These results to some extent supported the feasibility of AMR in the knee joint to be a predictor of functional outcomes in elderly $\mathrm{OA}$ patients after TKA.

For a long time, obesity has often been found to be associated with complications (shallow infection, deep infection, pulmonary embolism, etc.) in elderly OA patients following TKA [8, 9, 46, 47]. Using metaanalysis, Kerkhoffs et al. [8] and Si et al. [9] found that

Table 4 Simple and multiple linear regression of KSS total-post score

\begin{tabular}{|c|c|c|c|c|c|c|}
\hline & \multicolumn{3}{|c|}{ Simple linear regression } & \multicolumn{3}{|c|}{ Multiple linear regression } \\
\hline & $\beta$ & $95 \% \mathrm{Cl}$ & $p$ & $\beta$ & $95 \% \mathrm{Cl}$ & $p$ \\
\hline Age & 0.021 & -0.494 to 0.535 & n.s & & & \\
\hline BMl & -1.740 & -2.867 to -0.614 & 0.003 & -0.727 & -2.028 to 0.574 & n.s \\
\hline Sex & 0.153 & -4.924 to 5.230 & n.s & & & \\
\hline AMR & -6.817 & -10.134 to -3.500 & 0.000 & -5.573 & -9.564 to -1.582 & 0.007 \\
\hline Side & 3.159 & -1.867 to 8.814 & n.s & & & \\
\hline KSS total-pre & 0.069 & -0.052 to 0.189 & n.s & & & \\
\hline HSS-pre & 0.235 & -0.063 to 0.533 & n.s & & & \\
\hline
\end{tabular}

Cl confidence interval, n.s nonsignificant, BMI body mass index, AMR adipose to muscle area ratio, KSS score the Knee Society Score, HSS score the Hospital for Special Surgery knee score 
Table 5 Relationship between AMR and complications following TKA

\begin{tabular}{ll}
\hline & Complications $^{1}$ \\
\hline AMR 1st quartile (bottom 25\%) (0.85-1.83) & 0 \\
AMR 2nd quartile (1.83-2.27) & 0 \\
AMR 3rd quartile (2.27-2.72) & 0 \\
AMR 4th quartile (top 25\%) (2.72-5.56) & 3
\end{tabular}

${ }^{1} 1$ superficial incisional surgical site infection (SSI), AMR - 2.80; 1 urinary tract infection, AMR $-3.15 ; 1$ prosthesis failure (periprosthetic infection), AMR -3.63

the risk of both shallow and deep infections was higher in obese patients following TKA. DeMik et al. [46] and Sloan et al. [47] reported that the chance of postoperative complications would increase in obese patients following TKA. In our research, we used the new indicator-AMR - to evaluate obesity and found that patients with complications had higher AMR value of the entire study cohort (3rd quartile-4th quartile) (Table 5). Such an observation prompted that patients with high AMR might be more prone to postoperative complications.

\section{Limitations}

Our research has its limitations. First, the patients in our study had only functional outcome measurements. There was no physical and mental health assessment for patients following TKA due to the shortage of our database. However, KSS and HSS scores used in our research were representative in assessing the postoperative condition of TKA patients and could reflect the patients' clinical results well. Second, AMR in our study was a local parameter (surgical site). The muscle area in the measurement site only included part of the quadriceps (closely related to the knee joint). We failed to select the middle femur (the quadriceps were more developed) for research due to the limitations of knee MRI. Nevertheless, our research improved the measurement method of AMR by Dai et al. [22]. In our study, we measured AMR value in three levels of the knee (supra-patella, midline of the patella, joint line of the knee) and used their average as final AMR, with the hope to objectively reflect the local status of muscle and fatness of the knee. Third, the measurement and calculation of AMR were based on MRI, while MRI was not a routine examination before TKA in all patients. Nevertheless, as MRI had become a common examination in the diagnosis and treatment of knee joint diseases, this new indicator-AMR - could, to some extent, help doctors to predict the patient's prognosis and to make more precise treatment and rehabilitation plans. Finally, the number of samples was too small and the conclusions that originated from our research should be used with caution. It was important to point out that there were only 3 patients with postoperative complications, so that we could not use statistical methods to test the relationship between the AMR value and postoperative complications. However, based on the limited patients, we found AMR was a predictor of PROMS and might have a relationship with postoperative complications for the patients in our study. If there were more large-scale studies in the future, the effectiveness of AMR could be better verified.

\section{Conclusions}

In this study, the new obesity evaluation indicatorAMR, which was well related with BMI, was found to be a predictor of PROMS (KSS total-post score and HSSpost score) in elderly OA patients following TKA, while BMI was not. This suggested that AMR in the knee joint might be used as a stronger risk factor of functional outcomes in elderly OA patients after TKA more than BMI.

\section{Abbreviations}

AMR: Adipose to muscle area ratio; BMI: Body mass index; OA: Osteoarthritis; PROMS: Patient-reported outcome measures; TKA: Total knee arthroplasty; KSS score: The Knee Society Score; HSS score: The Hospital for Special Surgery knee score; MRI: Magnetic resonance imaging; SD: Standard deviations; SSI: Surgical site infection; CVA: Cerebrovascular accident; DVT: Deep vein thrombosis; IQR: Interquartile range; $C$ : Computed tomography; DXA: Dual-energy X-ray absorptiometry; BIA: Bioimpedance analysis; PJls: Prosthetic joint infections; PSD: Paraspinal muscle density; SMI: Skeletal muscle index; CSA: Cross-sectional area

\section{Acknowledgements}

Not applicable.

\section{Authors' contributions}

ZGL contributed to the study design. LCQ, CKM, and LJY collected the data. CJ, SJS, and HGY made the data analysis. ZGL and LCQ wrote the paper. CFY, WSQ, WYB, and XJ revised the manuscript. All authors read and approved the final manuscript.

\section{Funding}

This work was supported by grants from The Ministry of Science and Technology of China (2020YFC2002800).

Availability of data and materials

All the data are available in contact with the correspondence author.

\section{Ethics approval and consent to participate}

Informed consent was obtained from all the individual participants included in the study. This study was approved by the institutional review board of Huashan Hospital, Fudan University.

\section{Consent for publication}

Not applicable.

\section{Competing interests}

The authors declare that they have no competing interests.

Received: 20 May 2020 Accepted: 8 October 2020

Published online: 27 October 2020

\section{References}

1. Klaassen T, Alleleyn AME, van Avesaat M, et al. Intraintestinal delivery of tastants using a naso-duodenal-ileal catheter does not influence food intake or satiety. Nutrients. 2019;11(2):472. https://doi.org/10.3390/nu11020472.

2. Collaboration NCDRF. Trends in adult body-mass index in 200 countries from 1975 to 2014: a pooled analysis of 1698 population-based 
measurement studies with 19.2 million participants. Lancet. 2016;387(10026): 1377-96. https://doi.org/10.1016/S0140-6736(16)30054-X.

3. Misra D, Fielding RA, Felson DT, et al. Risk of knee osteoarthritis with obesity, sarcopenic obesity, and sarcopenia. Arthritis Rheumatol. 2019;71(2): 232-7. https://doi.org/10.1002/art.40692.

4. Chooi YC, Ding C, Magkos F. The epidemiology of obesity. Metabolism. 2019;92:6-10. https://doi.org/10.1016/j.metabol.2018.09.005.

5. Davison MJ, Maly MR, Adachi JD, et al. Relationships between fatty infiltration in the thigh and calf in women with knee osteoarthritis. Aging Clin Exp Res. 2017;29(2):291-9. https://doi.org/10.1007/s40520-016-0556-z.

6. Polat $A E$, Polat $B$, Gurpinar $T$, et al. The effect of morbid obesity (BMI $\geq 35$ $\mathrm{kg} / \mathrm{m}^{2}$ ) on functional outcome and complication rate following unicompartmental knee arthroplasty: a case-control study. J Orthop Surg Res. 2019;14(1):266. https://doi.org/10.1186/s13018-019-1316-5.

7. Ogden $\mathrm{CL}$, Fryar $\mathrm{CD}$, Hales $\mathrm{CM}$, et al. Differences in obesity prevalence by demographics and urbanization in US children and adolescents, 2013-2016. JAMA. 2018;319(23):2410-8. https://doi.org/10.1001/jama.2018.5158.

8. Kerkhoffs GM, Servien E, Dunn W, et al. The influence of obesity on the complication rate and outcome of total knee arthroplasty: a meta-analysis and systematic literature review. J Bone Joint Surg Am. 2012;94(20):1839-44. https://doi.org/10.2106/JBJS.K.00820.

9. Si HB, Zeng $Y$, Shen B, et al. The influence of body mass index on the outcomes of primary total knee arthroplasty. Knee Surg Sports Traumatol Arthrosc. 2015;23(6):1824-32. https://doi.org/10.1007/s00167-014-3301-1.

10. Sun $\mathrm{K}, \mathrm{Li} \mathrm{H}$. Body mass index as a predictor of outcome in total knee replace: a systemic review and meta-analysis. Knee. 2017;24(5):917-24. https://doi.org/10.1016/j.knee.2017.05.022.

11. Giesinger JM, Loth FL, MacDonald DJ, et al. Patient-reported outcome metrics following total knee arthroplasty are influenced differently by patients' body mass index. Knee Surg Sports Traumatol Arthrosc. 2018; 26(11):3257-64. https://doi.org/10.1007/s00167-018-4853-2.

12. Girardi FM, Liu J, Guo Z, et al. The impact of obesity on resource utilization among patients undergoing total joint arthroplasty. Int Orthop. 2019:43(2): 269-74. https://doi.org/10.1007/s00264-018-4059-8.

13. Shah NR, Braverman ER. Measuring adiposity in patients: the utility of body mass index (BMI), percent body fat, and leptin. PLoS One. 2012;7(4):e33308. https://doi.org/10.1371/journal.pone.0033308.

14. Cruz-Jentoft AJ, Baeyens JP, Bauer JM, et al. Sarcopenia: European consensus on definition and diagnosis: report of the European Working Group on Sarcopenia in Older People. Age Ageing. 2010;39(4):412-23. https://doi.org/10.1093/ageing/afq034

15. Babu JM, Kalagara S, Durand W, et al. Sarcopenia as a risk factor for prosthetic infection after total hip or knee arthroplasty. J Arthroplasty. 2019; 34(1):116-22. https://doi.org/10.1016/j.arth.2018.09.037.

16. Liu P, Ma F, Lou H, et al. The utility of fat mass index vs. body mass index and percentage of body fat in the screening of metabolic syndrome. BMC Public Health. 2013;13:629. https://doi.org/10.1186/1471-2458-13-629.

17. Brown MJC, Vella-Baldacchino M, O'Flaherty E, et al. Predicting patient reported outcome in total knee arthroplasty using body mass index and limb measurements. Knee. 2018;25(5):915-22. https://doi.org/10.1016/j.knee. 2018.06.015

18. Overgaard $A$, Lidgren $L$, Sundberg $M$, et al. Patient-reported 1-year outcome not affected by body mass index in 3,327 total knee arthroplasty patients. Acta Orthop. 2019;90(4):360-5. https://doi.org/10.1080/17453674.2019. 1604940.

19. Sveikata T, Porvaneckas N, Kanopa P, et al. Age, sex, body mass index, education, and social support influence functional results after total knee arthroplasty. Geriatr Orthop Surg Rehabil. 2017;8(2):71-7. https://doi.org/10. 1177/2151458516687809.

20. Visser AW, de Mutsert R, Loef M, et al. The role of fat mass and skeletal muscle mass in knee osteoarthritis is different for men and women: the NEO study. Osteoarthritis Cartilage. 2014;22(2):197-202. https://doi.org/10. 1016/j.joca.2013.12.002.

21. Watts CD, Houdek MT, Wagner ER, et al. Subcutaneous fat thickness is associated with early reoperation and infection after total knee arthroplasty in morbidly obese patients. J Arthroplasty. 2016;31(8):1788-91. https://doi. org/10.1016/j.arth.2016.02.008.

22. Dai AZ, Breite J, Pham H, et al. Adipose-to-muscle area ratio at the knee is superior to BMI in predicting post-operative outcome following arthroscopic meniscectomy. Arch Orthop Trauma Surg. 2019;139(3):355-60. https://doi.org/10.1007/s00402-018-3030-x.
23. Grotle M, Hagen KB, Natvig B, et al. Obesity and osteoarthritis in knee, hip and/or hand: an epidemiological study in the general population with 10 years follow-up. BMC Musculoskelet Disord. 2008;9:132. https://doi.org/10. 1186/1471-2474-9-132.

24. Maly MR, Marriott KA, Chopp-Hurley JN. Osteoarthritis year in review 2019 : rehabilitation and outcomes. Osteoarthritis Cartilage. 2019. https://doi.org/ 10.1016/j.joca.2019.11.008.

25. Insall JN, Dorr LD, Scott RD, et al. Rationale of the Knee Society clinical rating system. Clin Orthop Relat Res. 1989;248:13-4.

26. Insall JN, Ranawat CS, Aglietti $P$, et al. A comparison of four models of total knee-replacement prostheses. J Bone Joint Surg Am. 1976;58(6):754-65.

27. Mou P, Zeng Y, Pei F, et al. Medial femoral epicondyle upsliding osteotomy with posterior stabilized arthroplasty provided good clinical outcomes such as constrained arthroplasty in primary total knee arthroplasty with severe valgus deformity. Knee Surg Sports Traumatol Arthrosc. 2019;27(7):2266-75. https://doi.org/10.1007/s00167-018-5292-9.

28. Kwasny MJ, Edelstein Al, Manning DW. Statistical methods dictate the estimated impact of body mass index on major and minor complications after total joint arthroplasty. Clin Orthop Relat Res. 2018;476(12):2418-29. https://doi.org/10.1097/CORR.0000000000000493.

29. Pulido L, Parvizi J, Macgibeny M, et al. In hospital complications after total joint arthroplasty. J Arthroplasty. 2008;23(6 Suppl 1):139-45. https://doi.org/ 10.1016/j.arth.2008.05.011.

30. Poultsides LA, Triantafyllopoulos GK, Sakellariou VI, et al. Infection risk assessment in patients undergoing primary total knee arthroplasty. Int Orthop. 2018;42(1):87-94. https://doi.org/10.1007/s00264-017-3675-z.

31. Serbest $\mathrm{S}$, Tosun HB. Dislocation of a revision total knee arthroplasty: rare but serious complication. Pan Afr Med J. 2015;20:193. https://doi.org/10. 11604/pamj.2015.20.193.6374.

32. Tiftikçi U, Serbest S. Periprosthetic proximal medial femoral cortical destruction caused by a femoral arterial pseudoaneurysm. Clin Interv Aging. 2015;10:1967-70. https://doi.org/10.2147/cia.S97230.

33. Tosun HB, Uludağ A, Serbest $\mathrm{S}$, et al. A rare case of extensive diffuse nonpigmented villonodular synovitis as a cause of total knee arthroplasty failure. Int J Surg Case Rep. 2014;5(7):419-23. https://doi.org/10.1016/j.ijscr. 2014.04.031.

34. Oğurel T, Serbest $\mathrm{S}$, Oğurel R, et al. Blood chromium-cobalt levels in patients after total knee arthroplasty and their effect on the retinal nerve fiber layer and macular ganglion cell complex. Retina. 2020;40(6):1200-6. https://doi.org/10.1097/iae.0000000000002535.

35. Serbest S, Tiftikçi U, Karaaslan F, et al. A neglected case of giant synovial chondromatosis in knee joint. Pan Afr Med J. 2015;22:5. https://doi.org/10. 11604/pamj.2015.22.5.7481

36. Xu S, Chen JY, Lo NN, et al. The influence of obesity on functional outcome and quality of life after total knee arthroplasty: a ten-year follow-up study. Bone Joint J. 2018;100-B(5):579-83. https://doi.org/10.1302/0301-620X.100B5. BJJ-2017-1263.R1.

37. Singer SP, Dammerer D, Krismer M, et al. Maximum lifetime body mass index is the appropriate predictor of knee and hip osteoarthritis. Arch Orthop Trauma Surg. 2018;138(1):99-103. https://doi.org/10.1007/s00402017-2825-5.

38. Anker SD, Morley JE, von Haehling S. Welcome to the ICD-10 code for sarcopenia. J Cachexia Sarcopenia Muscle. 2016;7(5):512-4. https://doi.org/ 10.1002/jcsm.12147

39. Rossi FE, Lira FS, Silva BSA, et al. Influence of skeletal muscle mass and fat mass on the metabolic and inflammatory profile in sarcopenic and nonsarcopenic overfat elderly. Aging Clin Exp Res. 2019;31(5):629-35. https://doi. org/10.1007/s40520-018-1029-3.

40. Chang CD, Wu JS, Mhuircheartaigh JN, et al. Effect of sarcopenia on clinical and surgical outcome in elderly patients with proximal femur fractures. Skeletal Radiol. 2018;47(6):771-7. https://doi.org/10.1007/ s00256-017-2848-6.

41. Wagner SC, Sebastian AS, McKenzie JC, et al. Severe lumbar disability is associated with decreased psoas cross-sectional area in degenerative spondylolisthesis. Global Spine J. 2018;8(7):716-21. https://doi.org/10.1177/ 2192568218765399

42. Kavolus JJ, Sia D, Potter HG, et al. Saphenous nerve block from within the knee is feasible for TKA: MRI and cadaveric study. Clin Orthop Relat Res. 2018;476(1):30-6. https://doi.org/10.1007/s11999.0000000000000006.

43. Wu XD, Xiang BY, Schotanus MGM, et al. CT- versus MRI-based patientspecific instrumentation for total knee arthroplasty: a systematic review and 
meta-analysis. Surgeon. 2017;15(6):336-48. https://doi.org/10.1016/j.surge. 2017.06.002.

44. Dragoo JL, Johnson C, McConnell J. Evaluation and treatment of disorders of the infrapatellar fat pad. Sports Med. 2012:42(1):51-67. https://doi.org/10. 2165/11595680-000000000-00000.

45. Tiftikçi U, Serbest S, Burulday V. Can Achilles tendon be used as a new distal landmark for coronal tibial component alignment in total knee replacement surgery? An observational MRI study. Ther Clin Risk Manag. 2017;13:81-6. https://doi.org/10.2147/tcrm.S125551.

46. DeMik DE, Bedard NA, Dowdle SB, et al. Complications and obesity in arthroplasty-a hip is not a knee. J Arthroplasty. 2018;33(10):3281-7. https:// doi.org/10.1016/j.arth.2018.02.073.

47. Sloan M, Sheth N, Lee GC. Is obesity associated with increased risk of deep vein thrombosis or pulmonary embolism after hip and knee arthroplasty? A large database study. Clin Orthop Relat Res. 2019;477(3):523-32. https://doi. org/10.1097/CORR.0000000000000615.

\section{Publisher's Note}

Springer Nature remains neutral with regard to jurisdictional claims in published maps and institutional affiliations.

Ready to submit your research? Choose BMC and benefit from:

- fast, convenient online submission

- thorough peer review by experienced researchers in your field

- rapid publication on acceptance

- support for research data, including large and complex data types

- gold Open Access which fosters wider collaboration and increased citations

- maximum visibility for your research: over $100 \mathrm{M}$ website views per year

At BMC, research is always in progress.

Learn more biomedcentral.com/submissions 